\title{
MPC Based Trajectory Tracking for An Automonous Deep-Sea Tracked Mining Vehicle
}

\author{
Hongyun $\mathrm{Wu}^{1}$, Yuheng Chen ${ }^{2, *}$ a] and Hongmao Qin ${ }^{2}$ \\ ${ }^{1}$ Changsha Institute of Mining Research, Changsha 410000, China \\ ${ }^{2}$ Key Laboratory of Advanced Design and Manufacturing for Vehicle Body, College of Mechanical and Vehicle Engineering, Hunan \\ University, Changsha 410082, China \\ * Corresponding author: Yuheng Chen (chenyuheng@csu.edu.cn)
}

Manuscript Revised 04 Aug 2021; Accepted 06 Aug 2021; Published 23 Aug 2021

Academic Editor: Jinchao Chen 10

Abstract: Model predictive control (MPC) has been successfully used in trajectory tracking for autonomous vehicles based on certain kinematic model under low external disturbance conditions, but when there are model uncertainties and external disturbances, autonomous vehicles will fail to follow the pre-set trajectory. This paper studies trajectory tracking control based on MPC for an autonomous deep-sea tracked mining vehicle in polymetallic nodule mines with model uncertainty and external disturbances. A MPC algorithm is designed for trajectory tracking. To address model uncertainties caused by vehicle body subsidence and track slippage, a drive wheel speed correction controller is designed by experimental data fitting, and Kalman filtering (KF) and adaptive Kalman filtering (AKF) are introduced to improve tracking performance by rejecting external disturbances especially during curve tracking. To handle dead zones and obstacles during actual operation, an obstacle avoidance strategy is proposed that uses the tri-circular arc obstacle avoidance trajectory with an equal curvature for path re-planning. Finally, Simulink\&Recurdyn co-simulations validate the performance of the proposed MPC controller through a comparison with nonlinear MPC (NMPC).

Index Terms: Deep-sea tracked mining vehicle; Trajectory tracking; Model predictive control; Kalman filter; Path planning.

\section{Introduction}

the twenty first century, with the continuous depletion of land mines and the rising demand for fossil energy, the energy crisis is imminent. Fortunately, the oceans are rich in mineral resources, e.g., polymetallic nodules, cobalt-rich crusts, and polymetallic sulfides $[1,2]$. At present, the amount of marine resource storage already explored is hundreds of times than that on land, so deepsea mining is in urgent need of development.

The deep-sea mining system is an advanced intelligent transport system, as is shown in Fig. 1. A mining vehicle fell from a mother ship's surface support system through hose into the seabed for self-propelled operation. The surface support system mainly provides the surface operation support platform for the system and undertakes the deployment and recovery of underwater mineral collecting system and underwater conveying system, which mainly includes the mother ship, A-frame, steel frame, winch and positioning system, etc. The concentrator subsystem mainly collects polymetallic nodules on the seabed and crushes them into a suitable particle size, which mainly includes a traveling mechanism, a mineral collecting mechanism, a crushing mechanism, a hydraulic system, a measurement and control system and a navigation system, a positioning system, an acoustic imaging system, etc. The underwater conveying system including the relay station, hose, hard pipe, slurry pump, mainly conveys the crushed ore to the mother ship silo. The power conveying system contains power containers, cables and provides power for the underwater system.

In the area of rigid-body modeling and mechanical dynamics analysis of mining vehicles and operating systems, Dai built a mechanical simulation model of a mining vehicle based on the Adams/ATV module [3]. In this study, vehicle dynamics was ana- 
lyzed by taking into account the effects of multiple factors such as seabed hydrodynamics, track slippage, and soil shear, and a series of simulation was conducted on the mining vehicle in the longitudinal driving, steering, slope climbing, and ditch crossing cases. Liu et al. built a rigid-body model of a seabed mining vehicle and a surface mothership based on RecurDyn [4-6]. They analyzed the vehicle dynamics and horizontal and vertical displacements of each part of the deep-sea mining system through co-simulation experiments.

Since the terrain of polymetallic nodule mines is thin and soft, tracked mining vehicles are commonly used in polymetallic nodule mining areas to minimize the impact of subsidence slip. However, the slippage of tracks is inevitable and directly affects the driving performance of the mining vehicle. To address this issue, trajectory tracking has been widely studied in the literature.

In the area of mining vehicle trajectory tracking, $\mathrm{Li}$ et al. used fuzzy PID to control the speed of the left and right tracks to simulate the unilateral obstacle crossing condition when the vehicle drives in a straight line [7-9]. Numerical simulation was conducted using the point stabilization control theory. In practice, mining vehicles may not always operate on a pre-determined trajectory since they need to steer for obstacle avoidance and may also be trapped in some conditions. Therefore, more complex working conditions need to be considered. Moreover, these works mainly use the velocity and angular velocity at the center of mass as the control inputs, which may have negative impact on the control performance since the dynamic response of track drive wheels during slippage are neglected.

In recent years, model predictive control (MPC) has been widely used in trajectory tracking in the application of autonomous underwater vehicles (AUVs). An MPC motion controller was proposed in [10] for AUVs and a dynamic controller with an adaptive algorithm was further designed to improve the tracking accuracy. A predictive model controller based on the Lyapunov theory was proposed in [11] for AUV trajectory tracking. A detailed modeling procedure was designed and stability analysis was presented in the study, and the feasibility of the algorithm was verified through comparative simulation experiments. The modern control theory, together with intelligent algorithms, is increasingly being used to solve engineering problems $[12,13]$. The fusion of nonlinear model predictive control (NMPC) and sliding mode control can achieve better trajectory tracking performance and hence is widely used for AUV trajectory tracking [14]. To address model uncertainties in AUV trajectory tracking, an adaptive model predictive controller was designed in [15] with an extended system observer. Compared to traditional MPC, the proposed method improved trajectory tracking accuracy under the premise of stable algorithm convergence.

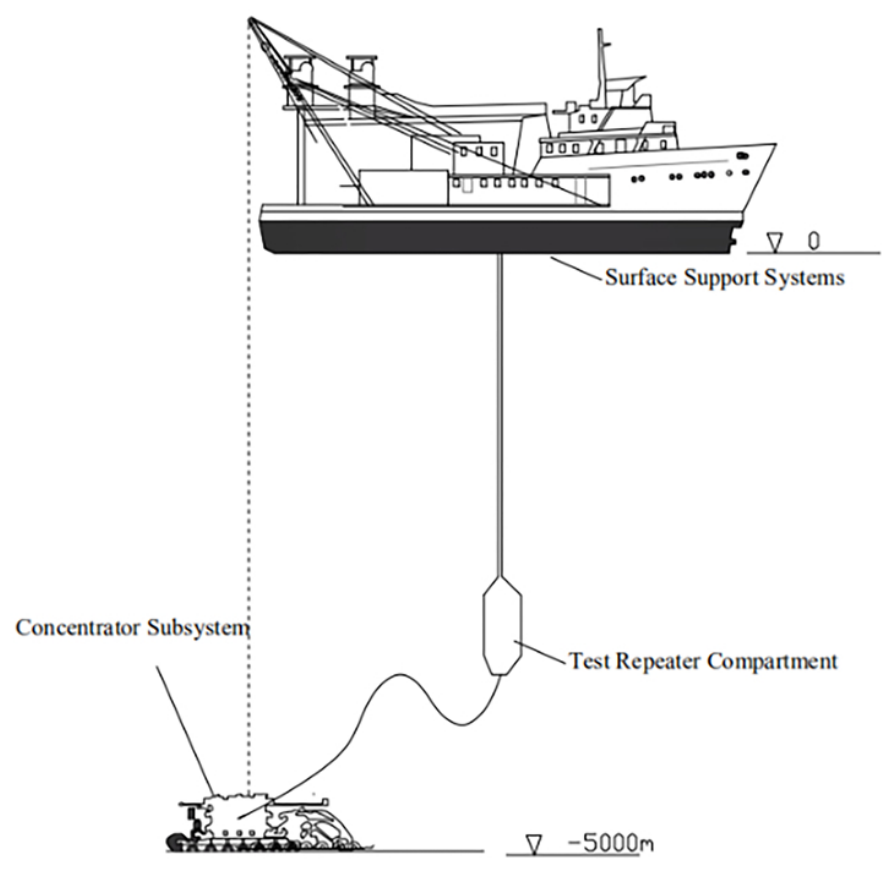

Fig. 1. Schematic diagram of the deep-sea mining system

In terms of automated driving, MPC is proven to be an effective trajectory tracking algorithm [16-18]. A hierarchical control strategy was proposed in [19] to improve the trajectory tracking performance. The control strategy firstly selects reasonable control points using sensor data, then generates trajectories using Bessel curve fitting, and finally uses NMPC for trajectory tracking. However, the trajectories generated in [20] may not always be smooth, since steering command is neglected. To ensure accuracy and trajectory smoothness during trajectory tracking, a control strategy, based on a quadratic programming method, was proposed in [21] for a four-wheeled vehicle by considering optimal steering. An MPC obstacle avoidance control strategy was proposed in [22] based on the Newtonian semi-smooth method to solve the quadratic programming for control of ground robots with good robustness and computing efficiency. Considering gasoline consumption, MPC is also used in [23] to optimize vehicle trajectories, with a new constraint introduced.

Note that MPC algorithms are subject to model mismatches, nonlinearity, and external disturbances. In recent years, the Kalman filter (KF) has been widely used to reduce the impact of disturbances [24]. Considering that the KF technique relies on a precise mathematical model, when there is uncertainty in the mathematical model, the adaptive Kalman filtering (AKF) technique can be used considering its better disturbance rejection 
performance than KF [25]. What's more, the emerging NMPC methods help improve tracking accuracy $[26,27]$, but the online computation takes too much time. To address this issue, this paper designs a trajectory tracking controller for a deep-sea tracked mining vehicle based on MPC. In this paper, we address the trajectory tracking problem for an automonous deep-Sea tracked mining vehicle. The contribution of this work includes:

1. An MPC based trajectory tracking algorithm is designed for a deep-sea tracked mining vehicle. A drive wheel speed correction controller is designed to ensure trajectory tracking accuracy considering model nonlinearities, vehicle body subsidence, and track slippage. The KF and AKF technique is incorporated into the MPC controller to address external disturbances including ocean currents. In the established soft seabed environment, the trajectory tracking performance of the proposed approach is validated through numerical simulation in the presence of model nonlinearities. Compared with NMPC [28, 29], the proposed approach has better computation efficiency at the cost of a slight reduction of tracking accuracy, which keeps a balance between control performance and computational complexity.

2. A trajectory re-planning layer is incorporated into the proposed MPC. Note that tracked vehicles can't change yaw angle as easily as four-wheeler vehicles, by introducing an equal-radius tri-circular arc trajectory for obstacle avoidance, the mining vehicle doesn't need to change yaw angle as frequently as it does when tracing trajectories generated by Bessel curves and quintuple polynomial fits $[25,26]$. The re-planning trajectory avoids the frequent change of yaw angles, which makes the deep-sea tracked mining vehicle easier and safer to avoid obstacles.

The rest of the paper is as follows. Section 2 introduces the kinematic model of the mining vehicle and the Kalman filter. Section 3 designs the MPC based trajectory tracking controller and the drive wheel speed correction controller. Section 4 presents the trajectory re-planning method for complex working conditions. Simulation results are given in Section 5, and conclusion is drawn in Section 6.

\section{Kinematic Model of the Mining Vehicle}

Denote the angular velocities of the left and right track drive wheels by $\omega_{1}$ and $\omega_{2}$, the coordinates of the vehicle by $(x, y)$, and the yaw angle of the center of mass by $\varphi$, as shown in Fig. 2.

Then we have

$$
\left\{\begin{array}{l}
v=\frac{v_{1}+v_{2}}{2}=\frac{\omega_{1}+\omega_{2}}{2} \\
\omega=\frac{v_{2}-v_{1}}{2}=\frac{\left(\omega_{2}-\omega_{1}\right) r}{B}
\end{array}\right.
$$

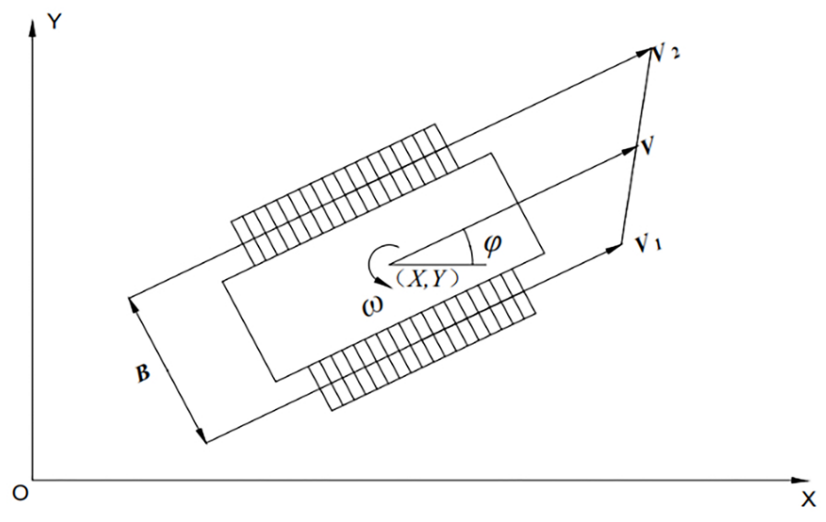

Fig. 2. Kinematics model of the mining vehicle

where $v$ is the speed of the center of mass, $\omega$ is the angular velocity, $r$ is the radius of the track drive wheel, and $B$ is distance between left and right tracks. Then the kinematics of the mining vehicle can be given as follows.

$$
\left[\begin{array}{l}
\dot{x} \\
y \\
\varphi
\end{array}\right]=\left[\begin{array}{l}
v_{x} \\
v_{y} \\
\omega
\end{array}\right]=\left[\begin{array}{c}
\cos \varphi \frac{\omega_{1}+\omega_{2}}{2} r \\
\sin \varphi \frac{\omega_{1}+\omega_{2}}{2} r \\
\frac{\left(\omega_{2}-\omega_{1}\right) r}{B}
\end{array}\right]
$$

\section{Trajectory Tracking Controller Design}

The trajectory tracking controller of the mining vehicle mainly consists of the following modules: an MPC controller, a Kalman filter, and a drive wheel speed correction controller, as shown in Fig. 3. In particular, The MPC controller uses the information of the tracking error to generate nominal angular velocities of the left and right track wheels $\bar{\omega}_{1}(t+1)$ and $\bar{\omega}_{2}(t+1)$, which will then be input into the drive wheel speed correction controller to generate the actual angular velocity $\omega_{1}(t+1)$ and $\omega_{2}(t+1)$.

Note that Kalman Filter is used to reduce the impact of disturbances and noise, and the MPC is used to generate nominal angular velocities. Then, by ensuring the stability of MPC and $\mathrm{KF}$, the stability of the proposed trajectory tracking controller can be guaranteed. Since the stability proof of MPC and KF has been given in [30] and [31], stability proof of the proposed controller will not be given in this paper.

\subsection{MPC Design}

Denote the predictive and control horizons by $N_{p}$ and $N_{C}$, respectively. By using Euler's method [32], the Jacobi linear approximation of Eq. (2) at each reference trajectory point is

$$
\tilde{X}(t+1 \mid t)=A_{t} \tilde{X}(t \mid t)+B_{t} \tilde{U}(t \mid t)
$$




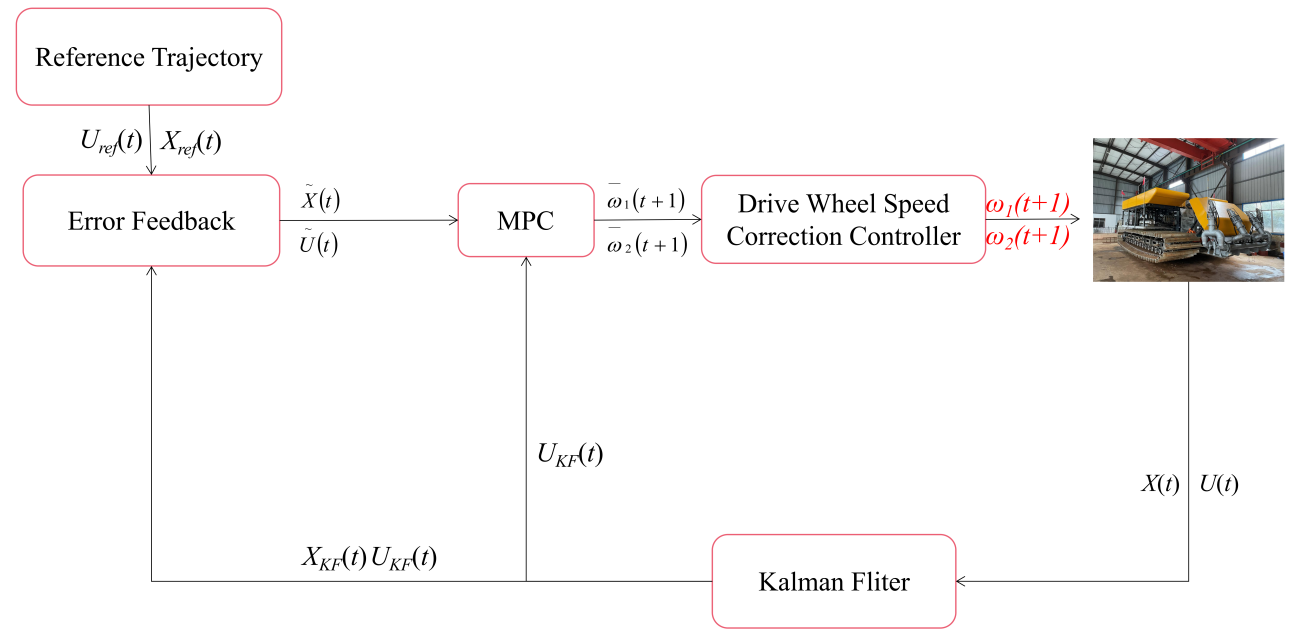

Fig. 3. Trajectory tracking control architecture of the mining vehicle

where

$$
A_{t}=\left[\begin{array}{ccc}
1 & 0 & -\frac{T r}{2}\left(u_{1 \text { ref }}(t)+u_{2 \text { ref }}(t)\right) \sin \varphi_{\text {ref }}(t) \\
0 & 1 & \frac{T r}{2}\left(u_{1 \text { ref }}(t)+u_{2 \text { ref }}(t)\right) \cos \varphi_{\text {ref }}(t) \\
0 & 0 & 1
\end{array}\right]
$$

$T$ is the sampling time.

$$
\begin{aligned}
& B_{t}= {\left[\begin{array}{cc}
\frac{T r}{2} \cos \varphi_{\text {ref }}(t) & \frac{T r}{2} \cos \varphi_{\text {ref }}(t) \\
\frac{T r}{2} \sin \varphi_{\text {ref }}(t) & \frac{T r}{2} \sin \varphi_{\text {ref }}(t) \\
-\frac{r}{B} T & \frac{r}{B} T
\end{array}\right] } \\
& \tilde{X}(t \mid t)=\left[\begin{array}{l}
x_{\text {ref }}(t)-x(t) \\
y_{\text {ref }}(t)-y(t) \\
\varphi_{\text {ref }}(t)-\varphi(t)
\end{array}\right] \\
& \tilde{U}(t \mid t)=\left[\begin{array}{l}
u_{1 \text { ref }}(t)-u_{1}(t) \\
u_{2 \text { ref }}(t)-u_{2}(t)
\end{array}\right]
\end{aligned}
$$

Equation (3) can be further rewritten as [29]:

$$
\begin{aligned}
\xi(t+1 \mid t) & =\tilde{A}_{t} \xi(t \mid t)+\tilde{B}_{t} \Delta u(t \mid t) \\
\eta(t \mid t) & =\tilde{C} \xi(t \mid t)
\end{aligned}
$$

where

$$
\begin{aligned}
\xi(t \mid t) & =\left[\begin{array}{c}
\tilde{X}(t \mid t) \\
\tilde{U}(t \mid t)
\end{array}\right], \tilde{A}_{t}=\left[\begin{array}{cc}
A_{t} & B_{t} \\
0_{2 \times 3} & I_{3}
\end{array}\right], \\
\tilde{B}_{t} & =\left[\begin{array}{c}
B_{t} \\
I_{3}
\end{array}\right], \tilde{C}=\left[\begin{array}{c}
I_{3} \\
0_{2 \times 3}
\end{array}\right]^{\mathrm{T}} .
\end{aligned}
$$

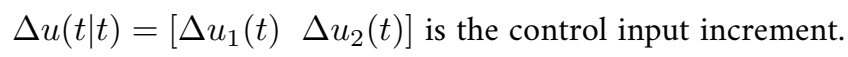

The predicted output of the system is expressed as follows:

$$
Y(t)=\Psi_{t} \xi(t \mid t)+\Theta_{t} \Delta U(t)
$$

where

$$
\begin{aligned}
& Y(t)=\left[\begin{array}{c}
\eta(t+1 \mid t) \\
\eta(t+2 \mid t) \\
\cdots \\
\eta\left(t+N_{c} \mid t\right) \\
\cdots \\
\eta\left(t+N_{p} \mid t\right)
\end{array}\right], \Delta U(t)=\left[\begin{array}{c}
\Delta u(t \mid t) \\
\Delta u(t+1 \mid t) \\
\Delta u(t+2 \mid t) \\
\cdots \\
\Delta u\left(t+N_{c} \mid t\right)
\end{array}\right] \\
& \Theta_{t}=\left[\begin{array}{cccc}
\tilde{C} \tilde{A}_{t} & 0 & 0 & 0 \\
\tilde{C} \tilde{A}_{t} \tilde{B}_{t} & \tilde{C} \tilde{B}_{t} & 0 & 0 \\
\cdots & \cdots & \ddots & \cdots \\
\tilde{C} \tilde{A}_{t}^{N_{c}-1} \tilde{B}_{t} & \tilde{C} \tilde{A}_{t}^{N_{c}-2} \tilde{B}_{t} & \cdots & \tilde{C} \tilde{B}_{t} \\
\tilde{C} \tilde{A}_{t}^{N_{c}} \tilde{B}_{t} & \tilde{C} \tilde{A}_{t}^{N_{c}-1} \tilde{B}_{t} & \cdots & \tilde{C} \tilde{A}_{t} \tilde{B}_{t} \\
\vdots & \vdots & \ddots & \vdots \\
\tilde{C} \tilde{A}_{t}^{N_{p}-1} \tilde{B}_{t} & \tilde{C} \tilde{A}_{t}^{N_{p}-2} \tilde{B}_{t} & \cdots & \tilde{C} \tilde{A}_{t}^{N_{p}-N_{c}-1} \tilde{B}_{t}
\end{array}\right]
\end{aligned}
$$

Here we introduced a relaxation factor $M$ to directly restrict control input increments and to guarantee the feasibility. The cost function is shown as follows:

$$
J(t)=\sum_{j=1}^{N_{p}}\|\eta(t+j \mid t)\|_{Q}^{2}+\sum_{j=1}^{N_{c}-1}\|\Delta u(t+j \mid t)\|_{R}^{2}+\rho M^{2}
$$

where $Q$ and $R$ are weight-matrices and $\rho$ is a weight coefficient.

Cost function Eq. (6) is transformed into the following form for quadratic programming.

$$
\begin{aligned}
J(\xi(t), \Delta U(t))= & {\left[\Delta U(t)^{\mathrm{T}}, M\right]^{\mathrm{T}} H_{t}\left[\Delta U(t)^{\mathrm{T}}, M\right]+} \\
& G_{t}\left[\Delta U(t)^{\mathrm{T}}, M\right]
\end{aligned}
$$

where

$$
H_{t}=\left[\begin{array}{cc}
\Theta_{t}^{\mathrm{T}} Q \Theta_{t}+R & 0 \\
0 & \rho
\end{array}\right], G_{t}=\left[2 X(t \mid t) Q \Theta_{t}, 0\right] .
$$


The control inputs and their increments are bounded as follows

$$
\begin{aligned}
& \Delta U_{\min } \leqslant \Delta U \leqslant \Delta U_{\max } \\
& U \_ \text {min } \leqslant D * \Delta U+U \leqslant U \_ \text {max }
\end{aligned}
$$

where

$$
D=\underbrace{\left[\begin{array}{ccccc}
1 & 0 & \cdots & \cdots & 0 \\
1 & 1 & 0 & \cdots & 0 \\
1 & 1 & 1 & \cdots & 0 \\
\vdots & \vdots & \ddots & \ddots & \vdots \\
1 & 1 & \cdots & 1 & 1
\end{array}\right]}_{N_{c} \times N_{c}} \otimes, \otimes \text { is the Kronecker product. }
$$

At the current moment $t$, the optimal control sequence $\Delta U(t)$ is output by addressing the following optimization problem.

$$
\begin{aligned}
& \min J(\xi(t), \Delta U(t)) \\
\text { s.t. } & \Delta U_{\min } \leqslant \Delta U \leqslant \Delta U_{\max } \\
& U \_\min \leqslant D * \Delta U+U \leqslant U \_\max
\end{aligned}
$$

The first element $\Delta u(t \mid t)$ of the optimal control sequence $\Delta U(t)$ will be used as the actual control input increment to generate the input for the drive wheel speed correction controller:

$$
\begin{aligned}
& u_{1}(t+1)=u_{1}(t)+\Delta u_{1}(t) \\
& u_{2}(t+1)=u_{2}(t)+\Delta u_{2}(t)
\end{aligned}
$$

When the mining vehicle is operating in a straight line and the heading angle of the preset trajectory is $0^{\circ}$, small angle assumptions can be made to eliminate the nonlinear terms $\sin \varphi_{\text {ref }}(t)$ and $\cos \varphi_{\text {ref }}(t)$ in Eq. (2). In this case, Eq. (2) can be rewritten as follows:

$$
\left[\begin{array}{c}
\dot{x} \\
y \\
\varphi
\end{array}\right]=\left[\begin{array}{c}
v_{x} \\
v_{y} \\
\omega
\end{array}\right]=\left[\begin{array}{c}
-\varphi_{\text {ref }} \frac{u_{2 \mathrm{ref}}(t)+u_{1 \mathrm{ref}}(t)}{2} r \\
\frac{u_{2 \mathrm{ref}}(t)+u_{1 \mathrm{ref}}(t)}{2} r \\
\frac{u_{2 \mathrm{ref}}(t)-u_{1 \mathrm{ref}}(t)}{B} r
\end{array}\right]
$$

and $A_{t}$ and $B_{t}$ in Eq. (3) can be rewritten as follows:

$$
\begin{aligned}
A_{t}= & {\left[\begin{array}{ccc}
1 & 0 & -\frac{T r}{2} \varphi_{\text {ref }}\left(u_{1 \mathrm{ref}}(t)+u_{2 \mathrm{ref}}(t)\right) \\
0 & 1 & \frac{T r}{2}\left(u_{1 \mathrm{ref}}(t)+u_{2 \mathrm{ref}}(t)\right) \\
0 & 0 & 1
\end{array}\right] } \\
B_{t}= & {\left[\begin{array}{cc}
\frac{T r}{2} & \frac{T r}{2} \\
\frac{T r}{2} \varphi_{\text {ref }}(t) & \frac{T r}{2} \varphi_{\text {ref }}(t) \\
-\frac{r}{B} T & \frac{r}{B} T
\end{array}\right] }
\end{aligned}
$$

\subsection{Kalman Filter Design}

Discrete model Eq. (1):

$$
\left\{\begin{array}{l}
X(i+1)=A X(i)+B U(i)+W(i) \\
Y(i)=C X(i)+V(i)
\end{array}\right.
$$

where the matrices $A$ and $B$, state $X$, and control input $U$ are consistent with Eq. (3). $C$ is the state observation matrix, and $W, \operatorname{Vin} R^{3}$ are uncorrelated process noise and observation noise, whose covariance matrices are $Q$ and $R \in R^{3}$, respectively.

The filtering process is as follows:

Prediction of the state:

$$
\hat{X}(i+1 \mid i)=A \hat{X}(i \mid i)+B U(i \mid i)
$$

Prediction of the covariance matrix:

$$
P(i+1 \mid i)=A P(i \mid i) A^{\mathrm{T}}+\Gamma Q \Gamma^{\mathrm{T}}
$$

Filter gain matrix updates:

$$
K(i+1)=P(i+1 \mid i) C^{\mathrm{T}}\left[C P(i+1 \mid i) C^{\mathrm{T}}+R\right]^{-1}
$$

Estimation updates:

$$
\left\{\begin{array}{l}
\hat{X}(i+1|i| i)=A X(i \mid i)+K(i+1) \varepsilon(i+1) \\
\varepsilon(i+1)=Y(i+1)-H \hat{X}(i+1 \mid i)
\end{array}\right.
$$

Covariance updates:

$$
P(i+1 \mid i+1)=\left[I_{n}-K(i+1) C\right] P(i+1 \mid i)
$$

To address model uncertainty caused by vehicle body subsidence, track slippage, and measurement noise, an AKF is introduced considering its ability to obtain more accurate state estimation when there are model nonlinearities [25]. Denote an update parameter $d k$ as follows:

$$
d_{k}=\frac{1-b}{1-b^{k}}
$$

where $b$ is the forgetting factor constant, whose value is between 0.95 and 0.99 . With the update parameter, the filter gain matrix updates in Eq. (16) can be rewritten as follows:

$$
\begin{aligned}
R(i+1)= & \left(1-d_{i+1}\right) R(i)+ \\
& \left(d_{i+1}[I-C K(i)] \varepsilon(i+1) \varepsilon(i+1)^{\mathrm{T}}\right) \\
& {\left.[I-C K(i)]^{\mathrm{T}}\right)+C P(i+1 \mid i) C^{\mathrm{T}} } \\
K(i+1)= & P(i+1 \mid i) C^{\mathrm{T}}\left[C P(i+1 \mid i) C^{\mathrm{T}}+R(i+1)\right]^{-1}
\end{aligned}
$$

Then the KF is modified into an AKF. Note that by introducing parameter $d_{k}$, the impact of accumulated noise from previous moments can be reduced, which hence increases the weight of the current moment and improves the robustness to model uncertainties. 


\subsection{Drive Wheel Speed Correction Controller Design}

Note that the track slippage of the mining vehicle, the instantaneous steering center change, track slippage and the body sinking caused by the shear force in polymetallic nodule mine are all influencing factors. Considering these factors, a drive wheel speed correction controller is designed to estimate the complex nonlinear relationship between the angular velocity of the left and right track wheels $\omega_{\text {track }}$ and the actual track travel speed $v_{\text {real }}$.

For the abovementioned influencing factors, define the track slippagage

$$
i_{i}=\frac{v_{\text {real }}-v_{\text {ref }}}{v_{\text {real }}}
$$

where $v_{\text {ref }}, v_{\text {real }}$ are the theoretical speed and actual speed of the track respectively. As for the other two factors, it is difficult to establish a mathematical model for them. Therefore, the design of wheel speed correction controller can be defined as the concept of incomplete identifiability.

Suppose that the maximum driving speed of the mining vehicle is $1.5 \mathrm{~m} / \mathrm{s}$, and there is a functional relationship $v_{\text {real }}=f\left(\omega_{\text {track }}\right)$ between $o m e g a_{\text {track }}$ and $v_{\text {real. }}$. To identify the function, a chassis test of the mining vehicle is conducted in the laboratory environment, as is shown in Fig. 4. In the test, a total of $m=8$ sets of drive wheel speeds are input into the mining vehicle and the corresponding actual track speeds are recorded. Then the function $v_{\text {real }}=f\left(\omega_{\text {track }}\right)$ is obtained by polynomial fitting as follows:

$$
\arg \min \sum_{i}^{m}\left(f\left(\omega_{\text {track }}(i)\right)-v_{\text {real }}(i)\right)^{2}
$$

where $f\left(\omega_{i}\right)$ and $v_{\text {real }}(i)$ are the $i$-th set of experimental data in the $m$ sets of data. The obtained function is

$$
f=0.001\left(-2.571 x^{4}+48 x^{3}+165 x^{2}+1858 x+41\right),
$$

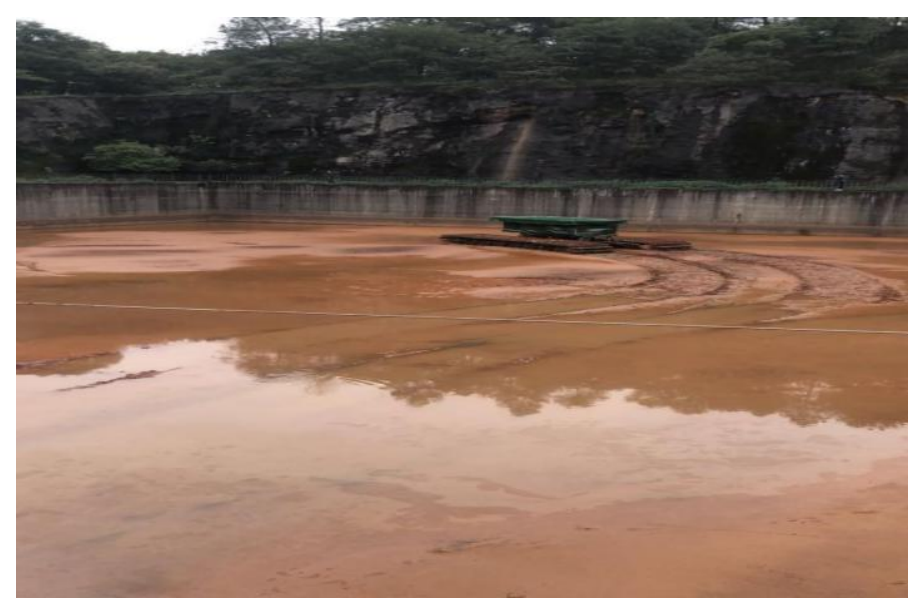

Fig. 4. Chassis test in the laboratory environment

and the fit of one track is shown in Fig. 5.

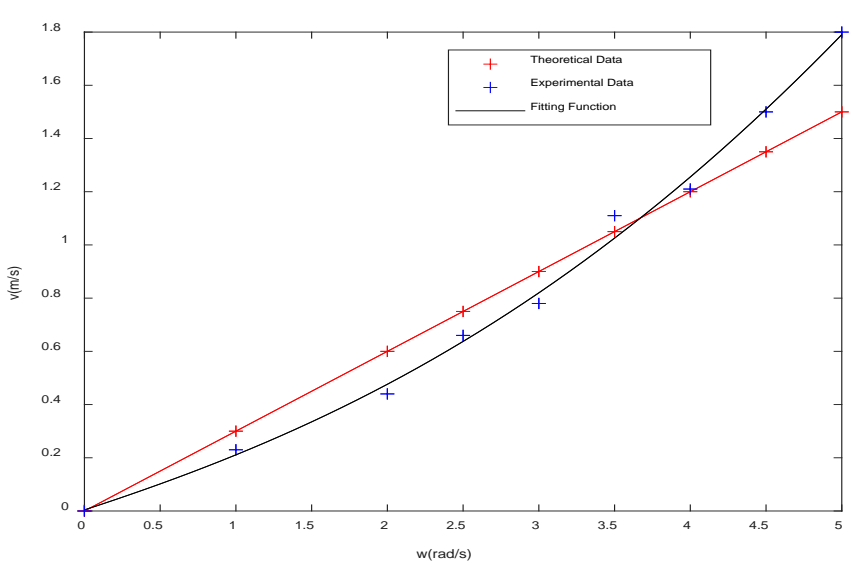

Fig. 5. Experimental data fit results

As shown in Fig. 3, the MPC controller outputs the desired angular velocities $u_{1}(t+1), u_{2}(t+1)$ of the drive wheels, and the desired speeds $v_{1}, v_{2}$ of the left and right tracks are determined by the kinematic model above. Finally, the drive wheel speed correction controller outputs the actual angular velocities $\omega_{1}(t+$ 1) $=f^{-1}\left(v_{1}\right), \omega_{2}(t+1)=f^{-1}\left(v_{2}\right)$.

\section{Trajectory Re-planning in Complex Conditions}

The seafloor of the polymetallic nodule mine site is soft and sparse, and the terrain is relatively flat. In seafloor modeling, slopes of no more than $10^{\circ}$ are marked as passable slopes, while slopes of more than $10^{\circ}$ are considered as obstacles. When the mining vehicle encounters a dead zone with impassable slopes on three sides of a pre-set trajectory, it needs re-plan a trajectory to back out of the area and drive around. This section presents trajectory re-planning of the mining vehicle in these cases.

\subsection{Trajectory Re-Planning for Obstacle Avoidance}

The generated obstacle avoidance trajectory should avoid the mining vehicle crashing into obstacles with minimum deviation from the pre-determined trajectory. In recent years, the Bessel curve or quintuple polynomial fit $[25,26]$ are widely used in obstacle avoidance. In these studies, yaw angles and the trajectory curvature need to be frequently changed in the control algorithm. The control inputs of a four-wheeled vehicle are the rear axle speed and the steering angle. Therefore, the frequent change of yaw angles and trajectory curvature can be realized by changing the four-wheeled vehicle's steering angles and the rear axle speed. However, for tracked vehicles, the control inputs are the angular velocities of the left and right track drive wheels, as shown in Eq. (2), which cannot be used directly to change heading angles and the trajectory curvature. The instantaneous curvature of the 
mining vehicle $\rho$ is given as follows:

$$
\rho=\frac{1}{R}=\frac{\omega}{v}=\frac{2\left(\omega_{2}-\omega_{1}\right)}{B\left(\omega_{2}+\omega_{1}\right)}
$$

where $r$ is the instantaneous turning radius.

Therefore, when the trajectory curvature $\rho$ changes, the left and right track drive wheels' angular velocities $\omega_{1}$ and $\omega_{2}$ need to be changed. From the analysis above, it can be seen that $\omega_{1}$ and $\omega_{2}$ need to be corrected online by the drive wheel speed correction controller to reduce the impact of model uncertainty caused by the track slippage and the instantaneous change of the steering center. Note that the drive wheel speed correction controller can reduce but cannot eliminate the track deviation. In this case, the deviation $\delta$ brought about by the model uncertainty can be shown as follows:

$\delta=\left[\begin{array}{l}\delta_{x} \\ \delta_{y}\end{array}\right]=\left[\begin{array}{l}\sum_{i=1}^{n} \sum_{t_{i}}^{t_{i+1}}\left(\frac{\Delta \omega_{1}(i)+\Delta \omega_{2}(i)}{2}\right) r \cos \varphi(i) \mathrm{d} t \\ \sum_{i=1}^{n} \sum_{t_{i}}^{t_{i+1}}\left(\frac{\Delta \omega_{1}(i)+\Delta \omega_{2}(i)}{2}\right) r \sin \varphi(i) \mathrm{d} t\end{array}\right]$

where $n$ is the number of times the angular velocities of the left and right track drive wheels change, and $\Delta \omega_{1}$ and $\Delta \omega_{2}$ are the deviations from the desired speed after processed by the drive wheel speed correction controller.

If the re-planned obstacle avoidance trajectory has variable curvatures, it will increase the complexity of the control algorithm and the trajectory deviation. Therefore, the re-planned obstacle avoidance trajectory should have less frequent curvature changes. As a result, Bessel curves or quintuple polynomial fit requiring real-time changes in curvature for obstacle avoidance trajectory are not applicable to the mining vehicle.

In this study, slopes that cannot be crossed by the mining vehicle are considered as obstacles. The slope projection is an irregular approximate circle. To simplify the obstacle model, the geometric center of the slope projection $O$ is selected as the center of the approximate circle, and the farthest distance of the projection boundary from the center of the circle is denoted by $R$, as shown in Fig. 6.

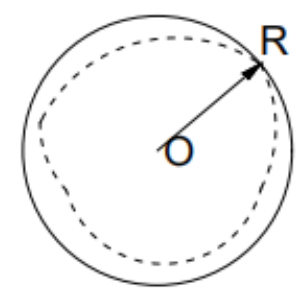

Fig. 6. Slope projection

From Eq. (24), we know that the deviation can be reduced by limiting the number of times the angular velocities of the left and right track drive wheels change. Denote the number as $n$. Based on the analysis, this study proposes the tri-circular arc obstacle avoidance trajectory with an equal curvature, as shown in Fig. 7. From Eq. (22), when the mining vehicle travels in a curve with an equal radius, the angular velocities of the left and right track drive wheels don't need to be changed. Therefore, the deviation of the obstacle avoidance trajectory can be reduced.

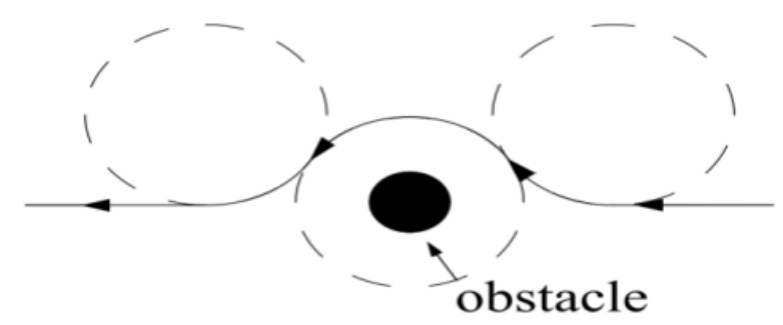

Fig. 7. Tri-circular arc avoidance trajectory

\subsection{Trajectory Re-Planning for Dead Zone Escape}

When the mining vehicle encounters a dead zone with impassable slopes on three sides of a pre-set trajectory, the operation's efficiency is affected since the vehicle needs to back out. To address this issue, this study views the entire dead zone as an obstacle, as shown in Fig. 9, so the mining vehicle can achieve dead zone escape by using the obstacle avoidance method designed above.

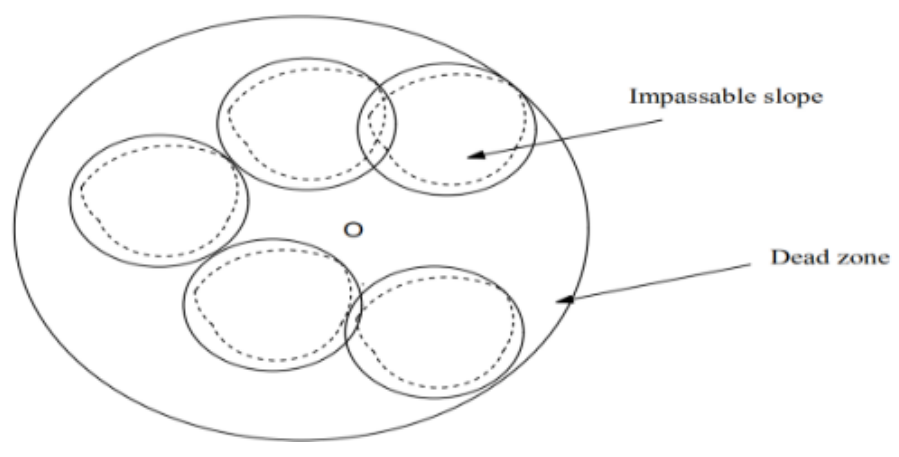

Fig. 8. Dead zone

\section{Simulation Verificaton}

This section verifies the proposed MPC tracking algorithm through RecurDyn \& Simulink co-simulation. The mechanical model of the mining vehicle is built in RecurDyn, as shown in Fig. 9. The model parameters are calibrated using the real mining vehicle, and are listed in Table I. The collection route of the mining vehicle is shown in Fig. 10.

During RecurDyn modeling, track surface contact parameters such as soil shear resistance Angle are set to simulate the terrain of the polymetallic nodules mining area, as shown in Fig. 11. 


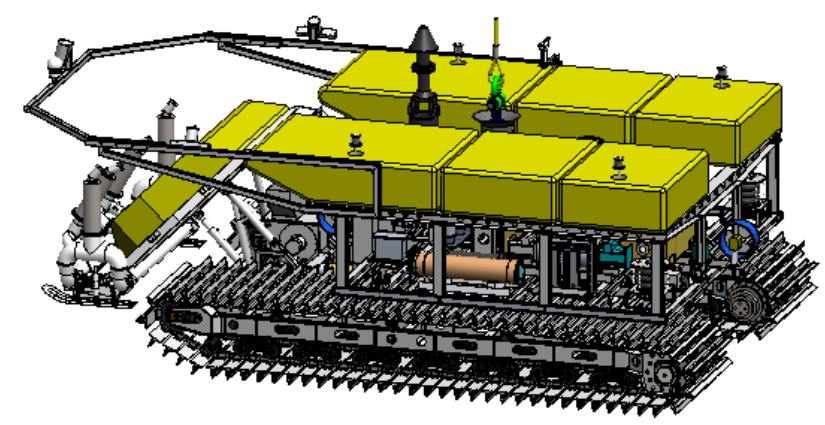

Fig. 9. Mechanical model of the mining vehicle

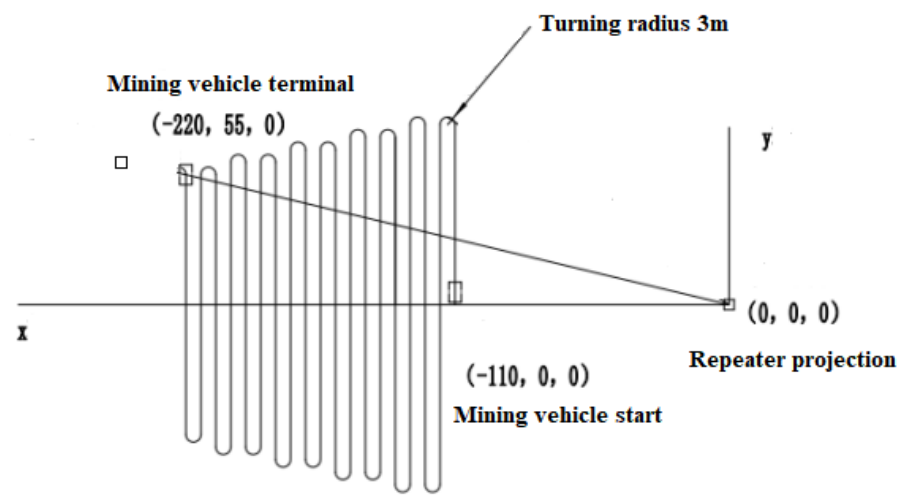

Fig. 10. Collection route for the polymetallic nodule mining vehicle

Table 1. Design parameters for the mining vehicle.

\begin{tabular}{ll}
\hline Design Parameter & Value hline \\
\hline Land Weight $(\mathrm{t})$ & 31 hline \\
Underwater Weight $(\mathrm{t})$ & 11 hline \\
Length $(\mathrm{mm})$ & 8800 hline \\
Width $(\mathrm{mm})$ & 4800 hline \\
Height $(\mathrm{mm})$ & 2950 hline \\
Drive Wheel Radius $(\mathrm{mm})$ & 300 hline \\
Maximum travel speed $(\mathrm{m} / \mathrm{s})$ & 1.5 hline \\
Land Weight $(\mathrm{t})$ & 31 hline \\
\hline
\end{tabular}

\subsection{Validation of the Drive Wheel Speed Correction Controller}

In this subsection, we validate the effectiveness of the proposed drive wheel speed correction controller. We conduct comparison of MPC with or without the proposed drive wheel speed controller, and the results are shown in Fig. 12.

As shown in Fig. 12 if the drive wheel speed controller is not introduced, the lateral error starts to increase when the mining vehicle tracks in the first circular trajectory. At the same time, the longitudinal position also does not match the reference position. Therefore, when the mining vehicle starts to track the second circular trajectories, the lateral error is further increased, due to the existence of caused by the instantaneous steering center change, track slippage and the body sinking. Moreover, the longitudinal position becomes more mismatched, ultimately leading to failure
Contact Parameter

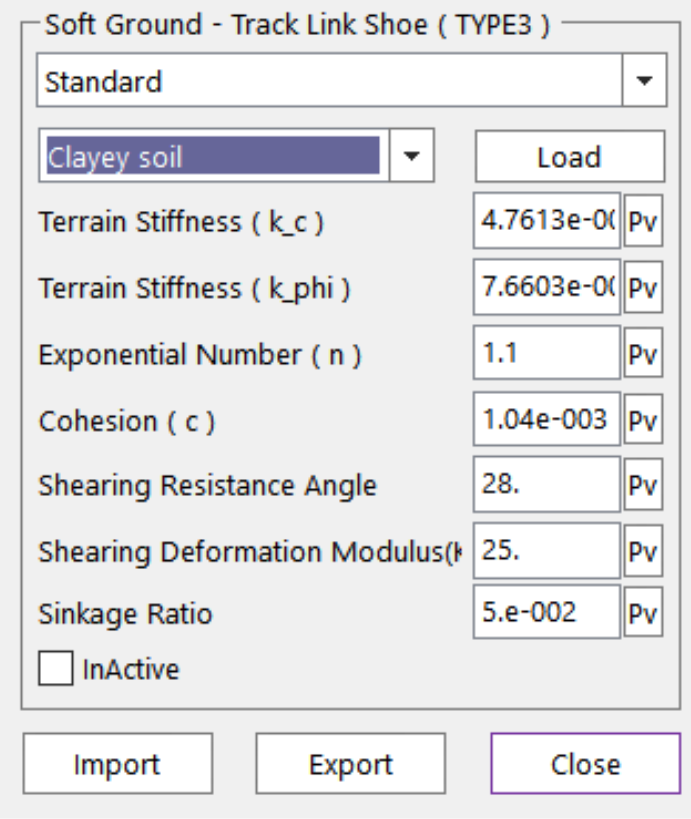

Fig. 11. Track contact surface parameters

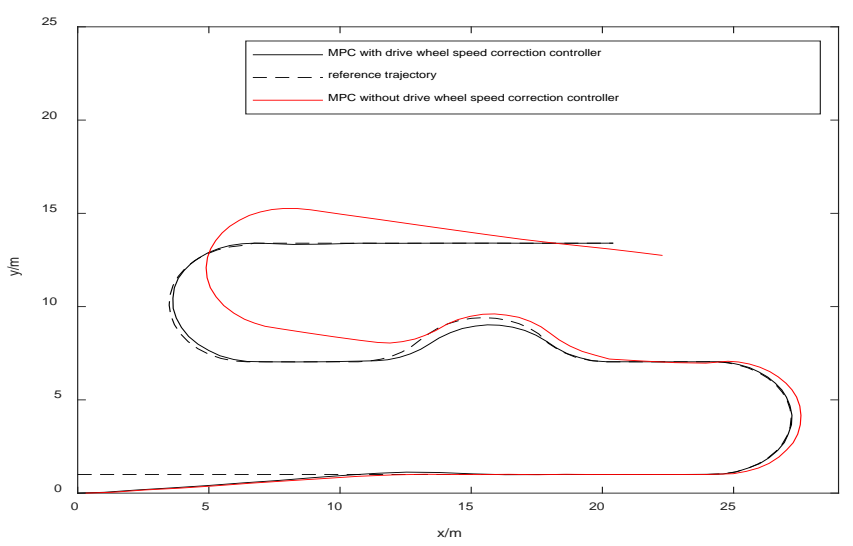

Fig. 12. Comparison of MPC with or without the drive wheel speed controller of trajectory tracking. In contrary, when the drive wheel speed correction controller is introduced, the mining vehicle can track the pre-set trajectory. This demonstrates the effectiveness of the drive wheel speed correction controller.

\subsection{Validation of the MPC Trajectory Tracking Controller}

This subsection validates the performance of the MPC based trajectory tracking controller in two cases: the presence of obstacles and the absence of obstacles in the pre-set trajectory.

The reference travel speed is set as $1 \mathrm{~m} / \mathrm{s}$. The reference trajectories are generated from 100 discrete sampling points, whose sampling period $T$ is 1 s. In particular, compared with MPC, MPC with KF and MPC with AKF are used to verify the tracking performance of the proposed controller. NMPC is used in comparative simulation experiment. In the test, the deviation is selected as the performance index, and it should not exceed $0.6 \mathrm{~m}$. The trajectory tracking computation times for 100 loops of con- 
trol cycles are also recorded in the simulation.

Case 1: Trajectory tracking without obstacles in the pre-set trajectory

The simulation results of trajectory tracking for Case I are shown in Fig. 13, Fig. 14 and Table II.

Table 2. MPC Trajectory tracking performance evaluation index.

\begin{tabular}{lll}
\hline Tracking control & Total computing time & Squared deviation (m) \\
\hline MPC & 313 & 0.184 \\
MPC with KF & 338 & 0.159 \\
MPC with AKF & 345 & 0.161 \\
NMPC & 574 & 0.141 \\
\hline
\end{tabular}

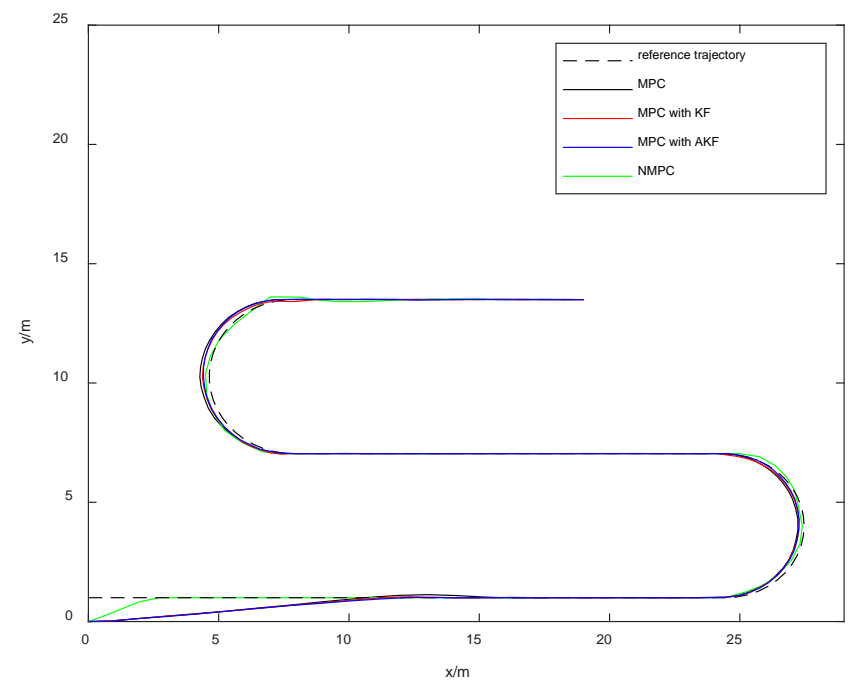

Fig. 13. Comparison of vehicle trajectories in the absence of obstacles

Figure 13 shows that NMPC can track straight lines faster, but MPC, MPC with KF, and MPC with AKF can all track the preset line trajectory. From Fig. 13, we know that MPC, MPC with $\mathrm{KF}$ and MPC with AKF get an ideal linear tracking performance, where the deviations can be negligible.

Figure 14 shows that the trajectory will deviate significantly from the pre-set trajectory if there is no filter in the MPC tracking controller during curve tracking. From Fig. 13, we know the maximum lateral deviation during curve tracking is about $0.35 \mathrm{~m}$, and the maximum deviation is about $0.42 \mathrm{~m}$. As a result, the trajectory is deviated from the pre-set trajectory, mainly caused by lateral deviation.

Figure 14 shows the maximum deviation of MPC with KF is reduced to about $0.35 \mathrm{~m}$, and the maximum lateral deviation is reduced to about $0.25 \mathrm{~m}$. In addition, the deviation of MPC with $\mathrm{AKF}$ is reduced to about $0.3 \mathrm{~m}$, and the maximum lateral deviation is reduced to about $0.25 \mathrm{~m}$. Both of them meet the tracking performance. Therefore, the filter can be introduced to improve the tracking performance of curve tracking by reducing the lateral deviation.
From Table II, it can be seen that the sum of deviation of MPC with AKF is a little less than that of MPC with KF, but the computing time of the AKF filter is little more than that of KF, about 7 s. Therefore, the tracking performance of MPC with AKF can be a little better than that of MPC of KF at the expense of computing time. Although NMPC's tracking accuracy can be better than the others, especially during curve tracking, the computation takes too much time, about $284 \mathrm{~s}$ more than MPC, which will result in poor real-time performance.

Case2: Trajectory tracking with obstacles in the pre-set trajectory

The simulation results of trajectory tracking for Case 2 are shown in Fig. 15, Fig. 16 and Table III.

Table 3. MPC trajectory tracking performance evaluation index.

\begin{tabular}{lll}
\hline Tracking control & Total computing time & Squared deviation (m) \\
\hline MPC & 407 & 0.221 \\
MPC with KF & 416 & 0.190 \\
MPC with AKF & 467 & 0.191 \\
NMPC & 683 & 0.159 \\
\hline
\end{tabular}

Figure 15 and Fig. 16 show that MPC, MPC with KF, and MPC with AKF have similar linear tracking performances.

From Fig. 15, we know MPC with AKF's trajectory is closer to the reference trajectory during first curving tacking. The tracking performance of MPC with AKF is a litter better than that of MPC and MPC with KF.

From Fig. 15 and Fig. 16, we know that after obstacle avoidance, compared to the MPC, MPC with KF and MPC with AKF's trajectories are even more deviated. The second curve tracking performance of MPC with AKF and MPC with KF is even a little worse than that of MPC.

The KF and AKF is based on the deduction of the Markov chain [33]. Each moment state is inevitably affected by the previous moment state. From the above analysis, it can be seen that despite limiting the number of angular velocity changes, the mining vehicle will inevitably suffer from model errors, caused by nonlinear terms in Eq. (2), affecting the estimation accuracy of the filter. If $\mathrm{AKF}$ or $\mathrm{KF}$ is introduced, the model errors will accumulate step by step during curve tracking in filter processing. Fortunately, small angle assumptions in line tracking, shown in Eq. (11), can gradually reduce the accumulated error of previous moments by eliminating the nonlinear terms. In contrast, if AKF or $\mathrm{KF}$ is not introduced, each moment state is more affected by the current moment error.

The distance of the mining vehicle going from obstacle avoidance to straight-line tracking and then to curve tracking is only about $5 \mathrm{~m}$, which may not be long enough to estimate the accu- 

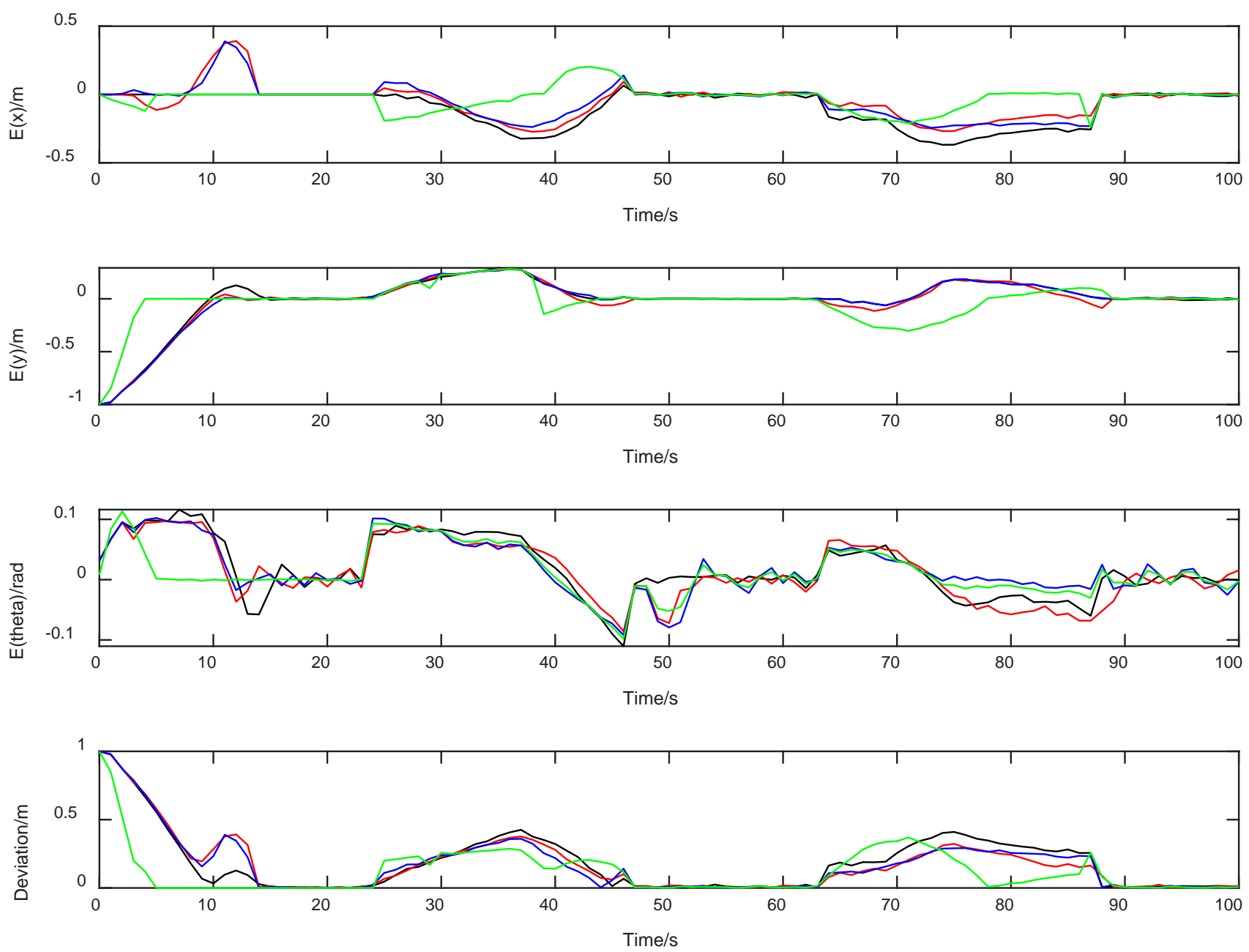

\begin{tabular}{|lll|}
\hline MPC & MPC with AKF & MPC with KF
\end{tabular}

Fig. 14. Comparison of tracking deviations in the absence of obstacles

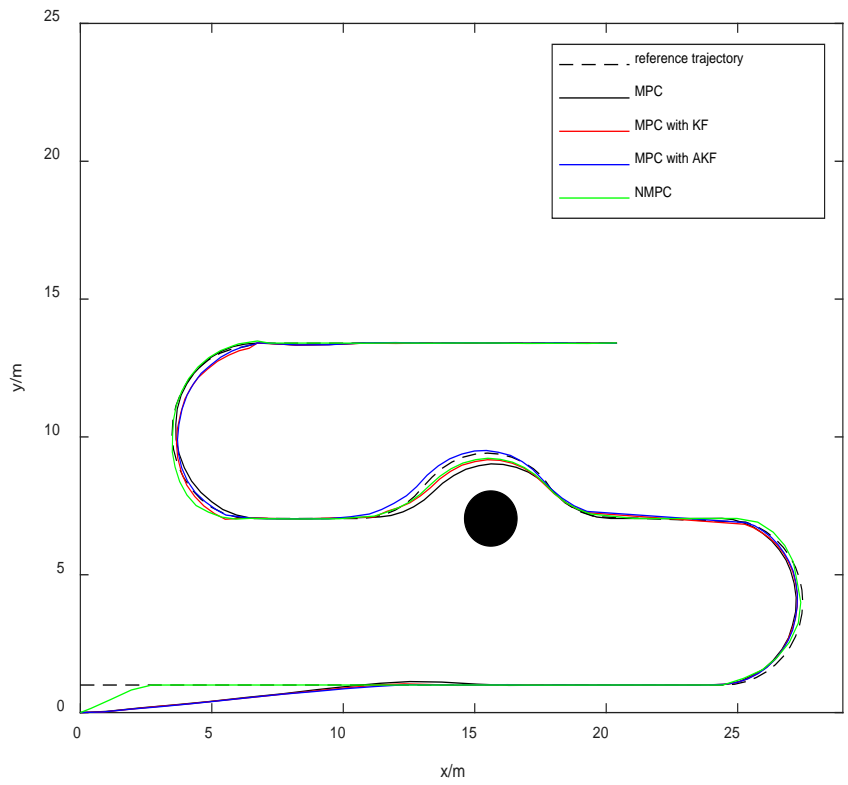

Fig. 15. Comparison of vehicle trajectories in the presence of obstacles mulated error of curve tracking in filter processing. Therefore, when KF or AKF is introduced, a long enough trajectory is required to eliminate the accumulated error after continuous curve tracking.

As shown in Table III, compared with MPC, both MPC with $\mathrm{AKF}$ and MPC with KF can improve trajectory performance to some extent. They are not as good as NMPC's which is more accurate during the entire trajectory, but both MPC with AKF and MPC with KF meet the accuracy requirement during the entire trajectory tracking. Note that a deviation of NMPC is $0.6469 \mathrm{~m}$, as is shown in Fig. 16, it fails to meet the accuracy requirement. Furthermore, the computation of NMPC also takes too much time, 216 seconds more than MPC. Therefore, considering the real-time requirements of actual operation of the mining vehicle, NMPC is not the suitable for trajectory tracking of the mining vehicle. 

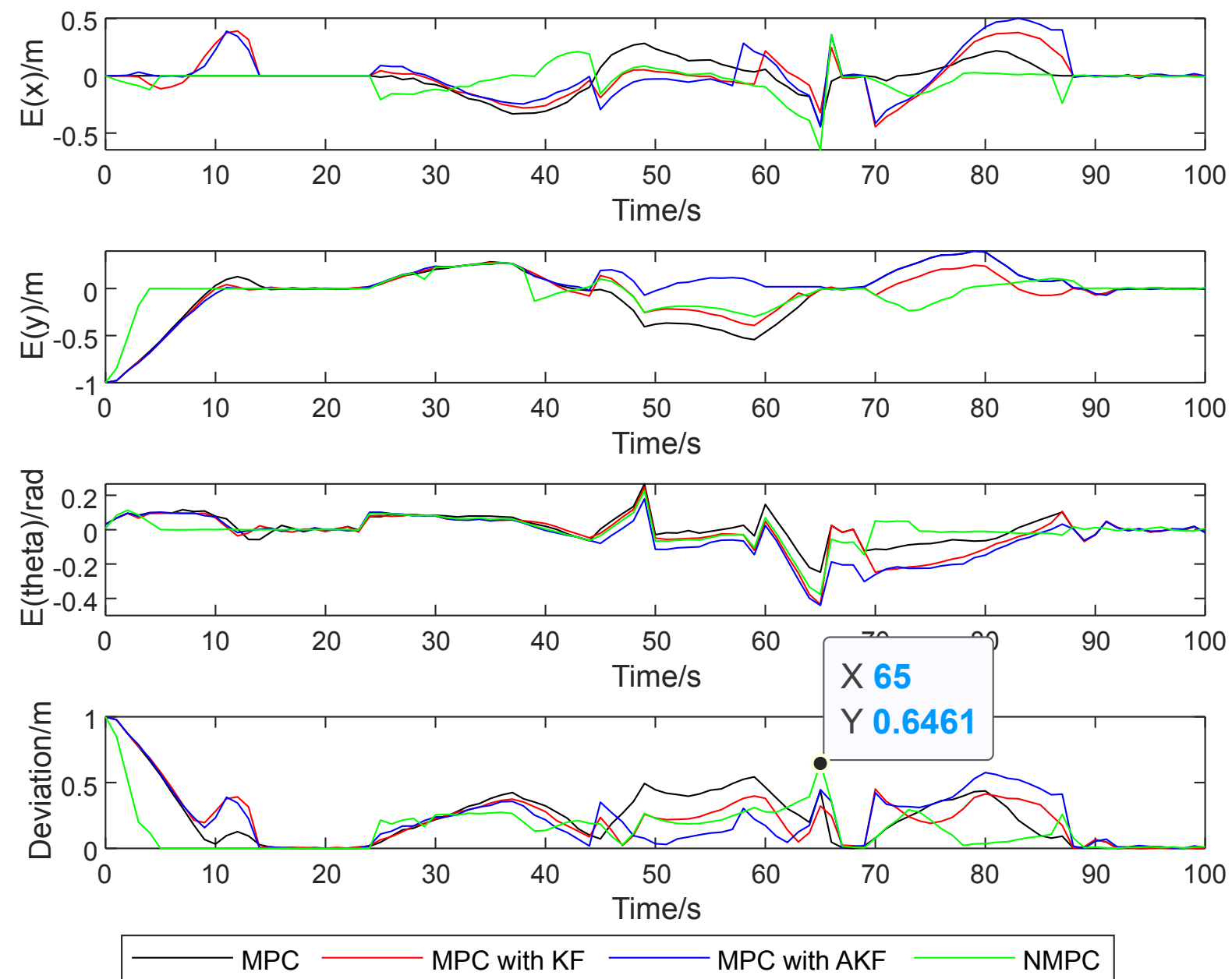

Fig. 16. Comparison of tracking deviations in the presence of obstacles

When the mining vehicle tracks an obstacle avoidance trajectory, it should avoid collision with obstacles. From Fig. 16 and Table III, the maximum lateral deviation of the MPC with AKF is even smaller than that of NMPC when avoiding obstacles. Furthermore, from Fig. 15, we know only MPC with AKF's trajectory doesn't deviate in the direction of the obstacle. Even if the computational time of MPC with AKF is 50 seconds more than that of MPC with KF, it is still acceptable compared to the computation of NMPC. Therefore, MPC with AKF's avoiding obstacle tracking performance is best.

\section{Conclusion}

This study has proposed a trajectory tracking controller of a mining vehicle using MPC. A drive wheel speed correction controller is incorporated into the controller to address slippage and model nonlinearity. The KF and AKF are introduced to improve the accuracy of curve trajectory tracking. Numerical simulation results demonstrate that the MPC with AKF is a little less accurate than NMPC but still meets the accuracy requirements and saves a lot of computation time. In the presence of obstacles, the tracking accuracy of the MPC with AKF is even better than that of NMPC.

\section{Acknowledgement}

This work was supported in part by the National Key Research and Development Project of China under 2016YFC0304102. Hongmao Qin's work was supported by the Key R\&D Program of Hunan Province, China under 2019GK2161.

\section{References}

[1] M. Lesage, C. Juliani, S. L. Ellefmo, "Economic Block Model Development for Mining Seafloor Massive Sulfides," Minerals, vol. 8, no. 10, 2018.

[2] S. E. Volkmann, T. Kuhn, F. Lehnen, "A comprehensive approach for a techno-economic assessment of nodule mining in the deep sea," Mineral Economics, vol. 31, no. 3, pp. 319-336, 2018.

[3] Y. Dai, S.-j. Liu, "An integrated dynamic model of ocean mining system and fast simulation of its longitudinal reciprocating motion," China Ocean Engineering, vol. 27, no. 2, pp. 231-244, 2013.

[4] Y. Dai, S.-j. Liu, "Theoretical design and dynamic simulation of new mining paths of tracked miner on deep seafloor," Journal of Central South University, vol. 20, no. 4, pp. 918-923, 2013.

[5] Y. Dai, S.-j. Liu, L. Li, "Dynamic Analysis of the Seafloor Pilot Miner Based on Single-Body Vehicle Model and Discretized Track-Terrain Interaction Model," China Ocean Engineering, vol. 24, no. 1, pp. 145-160, 2010. 
[6] J. Li, S. Liu, Y. Dai, "Effect of grouser height on tractive performance of tracked mining vehicle," Journal of the Brazilian Society of Mechanical Sciences and Engineering, vol. 39, no. 7, pp. 2459-2466, 2017.

[7] Q.-J. Han, S.-j. Liu, " Theoretical design and dynamic simulation of new mining paths of tracked miner on deep seafloor," Journal of Central South University, vol. 46, no. 02, pp. 472-478, 2015.

[8] L. Li, Z. Zheng, M. Chen, “ Point stabilization of seabed mining vehicle based on Lyapunov theory," Journal of Central South University, vol. 45, no. 08, pp. 2624-2628, 2014.

[9] L. Li, Y.-H. Zou, “ Tracking moving path of seabed mining vehicle based on theory of variable universe fuzzy control," Journal of Central South University, vol. 43, no. 02, pp. 489-496, 2012.

[10] W. Gan, D. Zhu, Z. Hu, et al., "Model Predictive Adaptive Constraint Tracking Control for Underwater Vehicles," IEEE Transactions on Industrial Electronics, vol. 67, no. 9, pp. 7829-7840, 2020.

[11] C. Shen, Y. Shi, B. Buckham, "Trajectory Tracking Control of an Autonomous Underwater Vehicle Using Lyapunov-Based Model Predictive Control," IEEE Transactions on Industrial Electronics, vol. 65, no. 7, pp. 57965805,2018

[12] Y. Chen, X. Xie, T. Zhang, et al., "A deep residual compensation extreme learning machine and applications," Journal of Forecasting, vol. 39, no. 6, pp. 986-999, 2020

[13] Y. Chen, C. Yi, X. Xie, et al., "Solution of Ruin Probability for Continuous Time Model Based on Block Trigonometric Exponential Neural Network," Symmetry-Basel, vol. 12, no. 6, 2020.

[14] R. M. Saback, A. G. Scolari Conceicao, T. L. Maia Santos, et al., "Nonlinear Model Predictive Control Applied to an Autonomous Underwater Vehicle," IEEE Journal of Oceanic Engineering, vol. 45, no. 3, pp. 799-812, 2020.

[15] B. Zhang, X. Sun, S. Liu, et al., "Adaptive model predictive control with extended state observer formulti-UAVformation flight," International Journal of Adaptive Control and Signal Processing, vol. 34, no. 10, pp. 1341-1358, 2020.

[16] X. Song, Y. Shao and Z. Qu, "A Vehicle Trajectory Tracking Method With a Time-Varying Model Based on the Model Predictive Control," in IEEE Access, vol. 8, pp. 16573-16583, 2020. doi: 10.1109/ACCESS.2019.2963291.

[17] H. Wu, Z. Si and Z. Li, “Trajectory Tracking Control for Four-Wheel Independent Drive Intelligent Vehicle Based on Model Predictive Control," in IEEE Access, vol. 8, pp. 73071-73081, 2020.doi: 10.1109/ACCESS.2020.2987812.

[18] S. Li, Z. Li, Z. Yu, B. Zhang and N. Zhang, "Dynamic Trajectory Planning and Tracking for Autonomous Vehicle With Obstacle Avoidance Based on Model Predictive Control," in IEEE Access, vol. 7, pp. 132074-132086, 2019.doi: 10.1109/ACCESS.2019.2940758

[19] A. Al-Mayyahi, A. A. Aldair, A. T. Rashid, "Intelligent Control of Mobile Robot Via Waypoints Using Nonlinear Model Predictive Controller and Quadratic Bezier Curves Algorithm," Journal of Electrical Engineering es Technology, vol. 15, no. 4, pp. 1857-1870, 2020.

[20] E. Kim, J. Kim, M. Sunwoo, "model predictive control strategy for smooth path tracking of autonomous vehicles with steering actuator dynamics," International Journal of Automotive Technology, vol. 15, no. 7, pp. 1155-1164, 2014

[21] A. Britzelmeier, M. Gerdts, "A Nonsmooth Newton Method for Linear Model-Predictive Control in Tracking Tasks for a Mobile Robot With Obstacle Avoidance," IEEE Control Systems Letters, vol. 4, no. 4, pp. 886-891, 2020

[22] Z. Yao, B. Zhao, T. Yuan, et al., "Reducing gasoline consumption in mixed connected automated vehicles environment: A joint optimization framework for traffic signals and vehicle trajectory," Journal of cleaner production, vol. 265, 2020.

[23] X. Yang, R. Seethaler, C. Zhan, et al., "A Model Predictive Contouring Error Precompensation Method," IEEE Transactions on Industrial Electronics, vol. 67, no. 5, pp. 4036-4045, 2020.

[24] C. Hide, T. Moore, M. Smith, et al., "Adaptive Kalman filtering algorithms for integrating GPS and low cost INS," in Plans 2004: Position Location and Navigation Symposium, pp. 227-233, 2004.
[25] G. Bai, L. Liu, Y. Meng, et al., "Path Tracking of Wheeled Mobile Robots Based on Dynamic Predicition Model," IEEE Access, vol. 7, pp. 3969039701, 2019.

[26] G. Bai, Y. Meng, L. Liu, et al., "A New Path Tracking Method Based on Multilayer Model Predictive Control," Applied Sciences-Basel, vol. 9, no. 13, 2019.

[27] P. Hang, S. Huang, X. Chen, et al., "Path planning of collision avoidance for unmanned ground vehicles: A nonlinear model predictive control approach," Proceedings of the Institution of Mechanical Engineers Part I-Journal of Systems and Control Engineering, 2020.

[28] L. Phan Gia, T. Nguyen Truong, "Real-Time Hybrid Navigation SystemBased Path Planning and Obstacle Avoidance for Mobile Robots," Applied Sciences-Basel, vol. 10, no. 10, 2020.

[29] C. Hong, Model Predictive control, Science Publishing House, Beijing, 2013.

[30] Z. Yan, P. Gong, W. Zhang, and W. Wu, "Model predictive control of autonomous underwater vehicles for trajectory tracking with external disturbances," Ocean Engineering, vol. 217, Dec 12020.

[31] Howard, S., Ko, H. L. and Alexan De R, W. E., "Parallel processing and stability analysis of the Kalman filter," IEEE Fifteenth International Phoenix Conference on Computers \& Communications. IEEE, 1996.

[32] OYELERE S S., "The application of model predictive control (MPC) to fast systems such as autonomous ground vehicles (AGV), “ IOSR Journal of Computer Engineering, vol.3, no. 3, pp. 27-37, 2014.

[33] Y. Ephraim, N. Merhav, "Hidden Markov processes," IEEE Transactions on Information Theory, vol. 48, no. 6, pp. 1518-1569, 2002.

\section{Biographies}

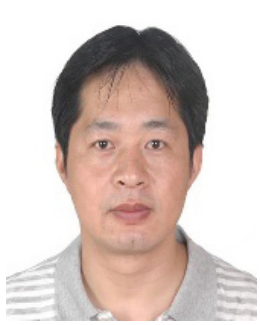

Hongyun Wu was born in Hubei, China in 1976. He is the director of the Institute of Marine Mining, Changsha Institute of Mining Research, Ph.D. in mechanical engineering, and a senior engineer. He is mainly engaged in the research of resource exploitation technology in the national long-term development project "International Regional Resource Development and Research". For more than ten years, he has been engaged in deep sea resource development research, and has successively presided over or participated in more than ten projects as a technical backbone, such as presiding over the "key technology research of 4-wheel drive self-propelled mining vehicle adapting to 6,000-meter class thin and soft substrate mining vehicle," "seabed mineral collection and navigation and positioning system development". He has written more than 20 scientific papers, including more than 10 EI-indexed ones. He has written more than 20 scientific research papers, of which more than 10 have been recorded by EI.

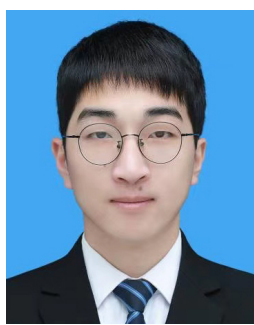

Yuheng Chen , M.S, was born in Suzhou, Jiangsu, China in 1996. He is an assistant Researcher in the School of Mechanical and Transportation, Hunan University, assistant director of Key Laboratory of Advanced Design and Manufacturing for Vehicle Body, College of Mechanical and Vehicle Engineering, Hunan University. 


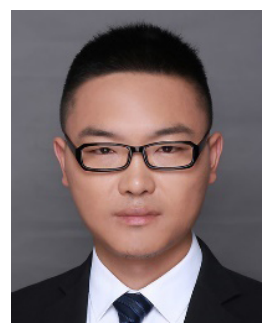

Hongmao Qin, Ph.D., is a research professorship in the School of Mechanical and Transportation, Hunan University, assistant director of Key Laboratory of Advanced Design and Manufacturing for Vehicle Body, College of Mechanical and Vehicle Engineering, Hunan University, and assistant director of Hunan Province Intelligent Transportation System Innovation Center. He is dedicated to the safety system architecture design of intelligent transportation system, specifically including the functional safety, information security and expected functional safety key technology research and development of intelligent driving system, intelligent dispatching system and intelligent interconnection system, and has carried out fruitful industrialization promotion work in the fields of intelligent mining system, intelligent marine system and intelligent express transportation system, and achieved good innovation progress. In recent years, he has published more than 30 academic papers, including $19 \mathrm{SCI} / \mathrm{EI}$ indexed papers, applied for/authorized 15 invention patents and 4 computer software copyrights; presided over/participated in more than 10 key projects such as National Natural Science Foundation of China, 863 Program, Science and Technology Support, etc., and undertook 4 national/industry/group standards, which have achieved good economic and social benefits. 